\title{
The Implementation of Clinical Decision Support System: A Case Study in Saudi Arabia
}

\author{
Sahar S. Alqahtani, Sabah Alshahri, Ahood I. Almaleh, Farrukh Nadeem \\ Department of Information Systems, Faculty of Computing and Information Technology, \\ King Abdulaziz University, Jeddah, Saudi Arabia. \\ E-mail: salqahtani0311@stu.kau.edu.sa,sama.alsabah123456@hotmail.com, aaaaa1591@hotmail.com, \\ fabdullatif@kau.edu.sa
}

\begin{abstract}
In recent years, the healthcare sector has shown inclination towards restructuring of healthcare systems to harmonize with technological innovations and adopting decision support system in routine clinical practices. The objective of this paper is to summarize challenges of Clinical Decision Support System (CDSS) and focus on the effectiveness of CDSS to improve clinical practice. This paper also describes the experience of CDSS in healthcare sector in Saudi Arabia and addresses the requirements for implementing successful CDSS with a real example. This study concludes that healthcare sector is in dire need to increase quality of patients' care and improve clinical practices by adopting CDSS.
\end{abstract}

Index Terms - CDSS in Saudi Arabia, the effectiveness of CDSS, challenges in CDSS, CDSS implementation.

\section{INTRODUCTION}

The health sector personnel including administration, physicians, nurses, and pharmacists rely heavily on patients' medical information. They consider it as a treasure to improve patients' healthcare and rapidly adapted to the latest technological tools that support patients' information storage and processing. The healthcare information systems support the healthcare environment to address the issues regarding the quality and cost of care.

Decision Support System (DSS) has been a part of healthcare information system for a long time. However, it supported only the financial or admin istrative domain. It has recently entered the domain of clinical data and clinical decision support system (CDSS) has emerged. The early CDSS is an extended form of previous expert system, where the goal is to create computer programs to simulate the human thinking by using machine-learning techniques. The machine learning is a class of computer algorithms that have the ability to understand human patterns of actions and intelligently use them to make decisions or forecast the future. The machine learning algorithms heavily rely on the available data from previous observations, which include information provided by the physician, staff, pharmacy and other healthcare individuals [1]. The health record entered electronically or registered manually by the clinicians is intelligently refined and presented to CDSS to enhance the quality of care in real time. The use of CDSS is rising in healthcare sector, especially in the organizations that suffer from problems with healthcare quality. The problems in healthcare quality are mainly due to the errors caused by human inattention and dangerous interaction between the treatments. CDSS can be effectively used to minimize such errors and enhance the patient's care quality.

The healthcare in Saudi Arabia has been significantly supported by the Saudi Government. Over the past few years, a rapid progress has been observed in the field of quality of care by the implementation of powerful CDSS techniques used in developed countries. In this paper, we briefly present different types of CDSS and the impact of CDSS in healthcare. We also summarize the challenges in implementation of CDSS. Then, we describe the require ments for successful imp lementation of CDSS. We also describe the use of CDSS in Saudi health sector. Further, we discuss the role of Saudi Ministry of Health in addressing healthcare issues in Saudi Arabia. Finally, we present literature survey of CDSS.

This paper organized as follows. Section II describes the related work. In Section III, we present different types of CDSS, followed by the impact of CDSS in healthcare in Section IV. We explain major challenges in implementation of CDSS in Section V. We describe main requirements for successful implementation of CDSS in Section VI. Section VII presents how CDSS is implemented in Saudi Arabia and the role of Ministry of Health to improve the clinical practices in Saudi Arabia. Finally, we conclude in Section VIII.

\section{RELATED WORK}

Many published studies discuss the CDSS effectiveness. The system and the diagnostic programs alert clinicians about the dangerous interactions of drugs. These programs can minimize the problems and errors, prevent complications and thus improve the clinicians' diagnoses. Early warning on the occurrence of damage may affect the quality of care and the cost involved [18, 19, 20, 48]. According to a study in England, the researchers found that implementing the computer-based guidelines can bring about a change in the health outcomes and the unanswered questions faced by the 
clinicians during the clinical encounter will offer a chance for using the CDSS [21, 22].

According to [23], they combined the four factors that were associated with implementing successful CDSS from various studies. The factors were 1) automating alerts and reminders, 2) providing suggestions at decision location and time, 3) offering actionable recommendations, and 4) computerizing the whole operation. These factors affect the process of care and handling of emergency situations. Decision making in emergency conditions in healthcare sector is discussed in [24], where the authors developed the emergency DSS frame work. A case study approach was used to show how fast the emergency decision support system deployed. The sharing of information is an important factor to implement the CDSS effectively [21, 24, 25].

In an article published in 2015 , there is a study in Malaysia on 12 public and private hospitals to determine the attitude of physicians in sharing their knowledge and understanding and to know their intention in adopting CDSS [25]. The findings indicate that following the advice given by CDSS, the clinicians assume that they lose their control on their work and lose specialized skills and knowledge where any non-professional can access to medical knowledge that is exclusive to the physician. So professional autonomy plays an important role in physicians' decision in adopting CDSS. In addition, this study improves 1) framework that encourages the decision maker to provide an environment to physicians to have efficient knowledge sharing and implement interactive CDSS, 2) the quality of services provided to the patient by using appropriate clinical IT systems in hos pitals.

Sittig et al. [6] discuss the critical need of high-quality CDSS to assist clinicians to do their work. They arranged the challenges depending on their importance. They classified the top challenges into three main classes; first class illustrates the concept of improving the efficiency of CDSS interference, second is creating new CDSS interventions, last one is related to distributing the interventions and knowledge of CDSS [6].

Jaspers and others argue that the EBM is a solution for reducing non-optimal patient care. They carried out literature search on renowned clinical libraries and analyzed high quality systematic reviews on CDSS. The objective is to conclude the effects of CDSS on the consequences of practitioners and outcomes of the patient. 52 out of 91 studies proved that CDSS had a positive effect on practitioners' performance (57\%) whereas 25 out of 82 studies showed its positive impact on patient outcomes (30\%) [7].

Sanchez et al. [26] discussed the main difficulties associated with CDSS. These are automation of the whole CDSS, integration in clinical workflow, extensibility and maintainability of the system, timely counselling, evaluation of effects and costs, and requirement for architectures that permit reusing and sharing of CDSS services and modules [26].

\section{TYPES OF CLINICAL DECISION SUPPORT SYSTEM}

There are various types of CDSS depending upon the organization framework, time of decision during the treatment, and to whom it is designed for (clinicians, nurses, pharmacists or staff). Basically, CDSS generates alerts based on patient's condition or responds to a clinician's input. Technically CDSS can be classified as a standalone system or part of the computer-based patient information system. The most general classification of CDSS is knowledge-based system (depends on mathematical foundation) or non-knowledge based system (apply machine learning approaches and mathematical foundation) [21].

\section{A. Knowledge-based system}

The most of the knowledge-based systems have emerged from the earlier research on expert systems, which intended to design computer programs to simulate human thinking in processing of data. It is widely believed that healthcare and patient care are among the most important fields to introduce knowledge-based systems to improve healthcare quality. The core of knowledge-based system is a database that contains clinical data. Every knowledge-based system contains three parts: knowledge-base, an inference engine, and a communication mechanism [2].

1. Knowledge-base: It contains a collection of information utilized by using if-then rules and the probability of diseases, sign, symptoms along with diagnoses and drug-drug interaction.

2. Inference engine: It integrates patient's current data with the information available from the knowledge base and makes suitable inferences about patient's condition.

3. Communication mechanis $\mathrm{m}$ : It is a way of how to get the outcome to the user by bringing the patient's data to the system to assist the user in making a decision. In the stand-alone system, the user enters the patient data into the system integrated with electronic medical record (EMR). The output is either in the form of an alert or some recommendations for taking the decision. For example, the user enters the sign and symptoms to the system or gets it from EMR. Then the inference engine integrates this information with that in the knowledge base. After that, the communication mechanism gives the output as a list of diagnoses to the user and the user selects the diagnoses [21].

\section{B. Non-knowledge-based system}

This type of CDSS uses machine-learning techniques as a kind of artificial intelligence that learns from experience by recognizing the pattern. The main types of non-knowledge-based system use genetic algorithm, and artificial neural network [3]. Artificial neural network learns from experiment to simulate human thinking. It consists of layer(s) of neurons, which are connected 
through weights. It has an input layer to get data and a hidden layer that processes the input data. The output layer provides result [13]. It has the ability to process the incomplete data and export the output so it does not need big database.

\section{THE IMPACT OF CDSS IN HEALTHCARE}

To deal with some deficiencies, healthcare organizations have started to adapt CDSS that helps the clinicians to make decisions depending on the evidence by providing them with recommendation in real time. [15]. There are more studies that discuss the impact of CDSS on clinical practices. Jaspers et al. [16] found 52 of 91 studies, which showed the positive impact of CDSS in preventive care and drug prescription.

There are studies, which discuss how the CDSS can significantly improve clinicians' performance by recommending the order, dosage and frequency of drug. In addition, CDSS contributes in improving the diagnostic accuracy and reducing the error, which consequently develops the workflow procedure to enhance the healthcare. It analyzes the patient's situation and provides recommendation to assist physicians in making the decision appropriate for the patient. CDSS also can create a plan to overcome the disease. It analyzes and matches the patient data with clinical database and generates a special treatment plan and thus gives clinician a way to improve the patient health and attain preferable outcomes. On the other hand, CDSS improves the clinical practices by cutting down the cost of care by using, for example, reminder system to avoid test repetition or avoid the drug-drug-interaction that may lead to extra treatment to handle the caused problems [17].

Implementation of CDSS provides an automatic support to clinician in decision making thus saving their time and improving quality of healthcare [15]. However, an effective implementation of CDSS in clinical practices must involve in-depth knowledge and training of clinicians.

\section{CHALLENGES IN CDSS IMPLEMENT ATION}

As healthcare organizations are moving towards CDSS era, some challenges have arisen. This part addresses the challenges that hinder the implementation of CDSS. These challenges mainly include issues regarding procedures, finance, society, and ethics.

\section{A. Alert Fatigue:}

The alert fatigue occurs when clinicians are encumbered with alerts and they turn off and ignore them. It is the source of continuing concern in CDSS [30]. Some clinicians set the alerts at a very high threshold i.e. for the most critical conditions only, considering the otherwise less important to be alerted about. Studies have proven that the clinicians do not take the advice of CDSS because of two reasons: overconfidence and difficulties to distinguish correct advice from incorrect one [31, 36].
Such practices restrict the utility of CDSS to full extent [31]. To use this system optimally, it is needed to refine the developed tools, which take electronic information of patient and based on this information provide suggestions and alerts, that may be followed by clinicians. If the clinicians understand the reasons behind the recommendations, they may accept them. So, the CDSS coupled with explanation services to provide rational and definitions of the recommendations may improve clinicians' confidence in the system [31, 73]. However, there is a need to filter the alerts provided by CDSS because turning off all alerts for avoid ing alert fatigue is a crude attempt [31,38]. Multilevel alert tiers approach has been recommended to avoid alert fatigue [38]. Also, approaches of context-sensitive decision support should be developed to handle too much data available in an erecord. CDSS should be available for review and operate in the background to alert the clinician in some situations or conditions i.e. the clinician may turn on all alerts for especially high-risk patients and leaving only the most dangerous alerts on for low-risk patients. The use of CDSSs should be less complex and redesigned to implement context-sensitive decision support, which may improve the result [31].

\section{B. Integration of Workflow:}

CDSS is complicated because it may use a wide range of information such as symptoms of patient, family history, genes history, as well as occurrence of disease trends [34]. A major challenge to CDSS implementation is workflow integration. Workflow has features, structure, and processes, which support clinical care. To improve the care, the workflow may need to be changed before, during or after the implementation of CDSS. The first step in the implementation process should be to analyze of workflow and identify the CDSS requirements. The clinicians should also be involved in the implementation. It has been recommended that they must also be trained to properly used CDSS [34]. Most of CDSS require the clinician to discontinue operating on their existing system, move to CDSS, enter the important information, and test the output [30]. CDSS needs to be flexible for all possibilities to fit the special requirements and a variety of workflows [29].

\section{Resistance to CDSS from patients and clinicians:}

Patients and clinicians may deny adopting CDSS because of the privacy and confidentiality issues [32, 35]. Furthermore, CDSS may also be rejected due to clinicians' reluctance to switch to a new system. Under pressure, the clinicians want to make quick decisions and record them in an easy and effective way without loss of much time. Whereas, with CDSS implementation, they may feel that it takes more time and their work is increased [29]. Also, the clinicians perceive knowledge sharing through CDSS as a threat to their professional autonomy [25]. Finally, the clinicians are also concerned about their relations with the patients when the CDSS becomes the third partner. Such concerns may not totally wrong in some cases, but should not to the point of 
refusal or resistance to the system that helps them to increase the healthcare cost and quality.

\section{Poor human interface:}

System interface is very important for clinical recommendation. The interface must be redesigned to improve the workflow and provide clear display of the recommendations and advice to facilitate the clinician work [6].

\section{E. Information filtered and integrated:}

The CDSS contains a large amount of patient data that must be filtered intelligently and presented briefly. Automatic summarization of data must provide the clinician a clear view about the patient's condition from previous visit and treatment. Also, the information in healthcare is dispersed and there is no rule or standard to integrate the data [47]. A large clinical database is thus needed to improve the CDSS [6].

\section{F. CDSS is regarded an expensive project:}

High cost of CDSS includes costs of adoption, implementation, continuing maintenance, support, and training required after CDSS installation [33]. The high costs of CDSS adoption and implementation come from the installation and purchasing of hardware and software devices like laptops or personal computers, scanners, and printers. The CDSS adoption and implementation costs also include cost of end-users' train ing [33]. The ongoing maintenance of CDSS is also costly because sometimes hardware needs to be changed and software needs to be upgraded. However, with increased adoption of CDSS in recent years the systems costs have descended drastically. Therefore, managers should reconsider the costs of software maintenance and equipment upgrade [32, 39].

\section{G. Standard of Care:}

The 'standard of care' is a term that describes the steps clinicians should take to provide appropriate care for the patient [40]. The clinicians are responsible to do the best for patient care and should learn how to meet this responsibility. Any healthcare information system is supposed to have an important responsibility, but sometimes there is a negative side the clinician must avoid [27]. The risk avoidance is an ethical duty of clinicians that they must do [40]. This requires a suitable approach to motivate the clinicians for proper use of CDSS to avoid growing risk of patients. A well-known approach to do this is called "progressive caution". The progressive caution encourages use of CDSS by taking its core values with suitable oversight and caution levels [41, 46].

\section{H. Appropriate Use and Users:}

The appropriate use of CDSS refers to its use only for purposes it is developed for. For example, if a system is designed for learning students, it cannot be relied on for CDSS. Secondly, use CDSS with a sufficient practicing. The key requirements for using CDSS are proper training and distinguishing capabilities for utilizing the system [44,
45]. Ethical concerns emerge due to uncertain training levels and inadequate capabilities of users [28]. The third ethical concern is its suitable utilization in practice assessment, quality evaluation and so forth. There are insufficient reasons to set a policy to prefer decisions recommended by CDSS [41].

\section{Professional Relationship:}

Recently, an idea of "engage in decision making" emerged where the patients and care providers are considered as participants. This idea emerged due to the fact that the patients have mistakenly been recognized as unaware and that the physicians have knowledge of everything [42]. For this, computer as a third partner has been introduced. Two ethical concerns rise here. First, a conceptual distance will be created by the computer between patient and provider. Some patients and providers take a view that a computer output is more accurate and correct than human output. This view produces destruction into shared decision-making. Whereas, the computer can present a diagnosis, which can increase the probability of misleading a patient's decision [41]. Right now, the clinician is supposed not to reveal to a patient that CDSS was utilized as a part of his or her state. But here the second ethical is sue appears as to whether patients ought to be given this information. It must be determined that the patient has an understanding of medical information and clinician can understand strategies and connections. In any situation, the use of computer data to trump patients is inappropriate [43]. Here, an issue arises frequently as to give patients an access to CDSS and make them demand from clinicians or criticize or challenge them. Over recent years, there was a slant to the use of CDSS by patients though it is inappropriate and dangerous. A clinician should make clear that CDSS is not an alternative to professionals in health sector and that the clinical practices require more than statistical induction from lab values and others [41].

\section{THE REQUIREMENT S FOR SUCCESSFUL IMPLEMENT ATION OF CDSS}

The recommendations and requirements can be classified into three categories as shown in figure 1 . The first requirement is related to the clinical guidelines about the involved CDSS content and knowledge. The second group of requirements is concerned with incorporating CDSS and Hospital Information System (HIS), corresponding to clinical workflow practice and adopting a mechanism of active and passive alert. Lastly, the remaining requirements related to the quality of advice provided by CDSS as output.

\section{A. The contents of CDSS}

\section{- The clinical contents must be right}

The hospital's clinical protocols, rules, and guidelines have to be converted into computerized formats to be incorporated with HIS that can support medical evidence. In fact, this is very difficult because the clinical protocols, 
rules, and guidelines are usually generalized and not specific for patients that make this is sue very complicated [7]. To overcome this challenge, the hospital should assign multidisciplinary experts to develop the reliable clinical rules.

- The clinical contents must be reliable

The major situations, which decrease or weaken such system's value, are mainly the unclear information content, the minimum alert specificity, very generalized, and a high sensitivity of the system [12]. So, to as sure the accuracy of alerts and messages and guarantee the validity of clinical rules/guidelines, the contents should be tested during computerizing the clinical rules/guidelines.

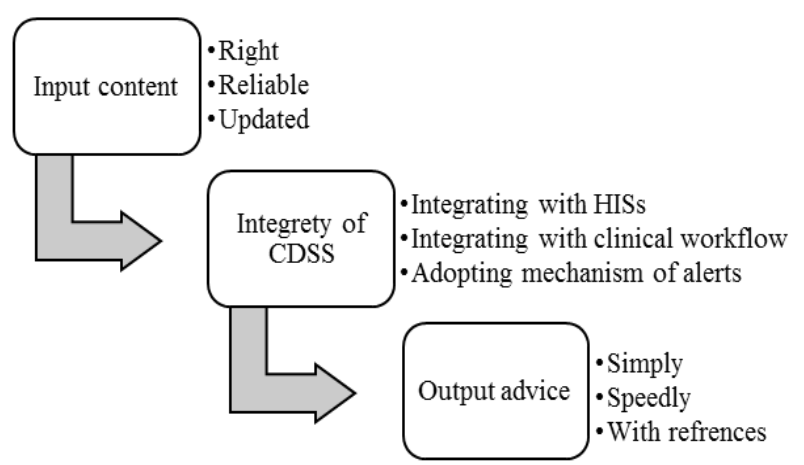

Fig.1. Requirements of CDSS success

- The clinical content must be updated

CDSS rely on knowledge of clinical that is in a case of continuous change [10]. So there is needed to establish a mechanism for reviewing and updating clinical knowledge related to rules and guidelines in order to maintain the effective use of CDSS.

\section{B. The clinical advice of CDSS}

- Simple to recognize and respond

The effectiveness of CDSS lies on delivering messages, which are easy to understand and respond and thus reduce the time and effort required by clinicians to respond to the system advice [12, 6, 7]. The developers of such system must foresee the need of users for interpreting and summarizing data into actionable advice.

\section{- High speed and low time}

To gain user satisfaction and acceptance, the computer systems should be of high speed to response quickly [8]. Therefore, CDSS advice and recommendations have to be obtainable to the clinician at the right point in a timely manner in the process of decision-making.

\section{- Advice with references}

Clinicians usually want to know more about specific topics relative to new evidence or practices in their expertise domain [9]. To construct more confidence in the accuracy, veracity, and credibility of the CDS system, they should provide the source of information that they have with the provided advice.

\section{Integrity of CDSS}

- $\quad$ DSS must be integrated with clinical workflow

One of the factors for success is to make CDSS appropriate for integration in normal and regular clinical workflow [12]. The perfect understanding of clinical workflow and user expectations/needs will have a very positive effect on CDSS success.

- $\quad$ CDSS must be integrated with HISs

It is very difficult for clinicians to find enough time from their routine activities to enter each patient data in CDSS on regular basis [6]. With integration of CDSS with HIS especially with electronic health records (EHR) will provide pertinent information to clinicians at a suitable time [12]. This integration can guarantee the acceptance of CDSS by clinicians.

- $\quad$ Active and passive mechanisms of CDSS alert

The alerts are classified into three levels: critical, moderately important and least important [12]. The critical alert is an active alert, where clinicians are informed by CDSS to make decision and take prompt action. While for least and moderately important, a passive alert is sent to clinicians, where they need to inquire about the correct recommendations to follow [12].

In nutshell the following are the main requirements for successful implementation of CDSS:

1. To embrace expert clinicians from start in the process of development of clinical protocols, principles and guidelines

2. To raise the acceptance of these rules and enhance user commitment where these expert clinicians are the end users of the system.

Implementing the system in clinical practices will encourage other users to utilize and accept such system. Clinicians find it challenging to invest effort and time in using and learning computer systems $[12,6]$. With these strategies, CDSS can help clinicians by providing associations and links between various data fields to enable clinicians to navigate between them.

\section{IMPLEMENT ATION OF CDSS IN SAUDI ARABIA}

The CDSS has become an important Evidence-Based Medicine (EBM) tool to improve safety of patients. However, it requires obligation to guidelines for treatment and prevention and to avoid errors in medication. CDSS provides clinicians with the desired knowledge of specific disease or patient. According to the 
department of medical informatics in the hospital of King Faisal Specialist and Research Center in Riyadh, Information Technology (IT) worked on determin ing best strategies and factors for successfully manage CDSS knowledge $[12,13]$. In the following, we will discuss the role of Ministry of Health $(\mathrm{MoH})$ to improve the clinical practices and some examples of CDSS implementation in Saudi Arabia.

\section{A. Example of CDSS implementation in Saudi Arabia}

In emergency department, there is a critical need to make the decision quickly and accurately to save the life in real time [14]. The emergency physicians face some serious and sensitive situations of diseases. They do not have adequate knowledge to deal with all aspects of diseases. So the emergency department needs some guidelines to define the quality of care and provide support to the physician to make a decision by establishing computerized clinical practices guideline (CPG). In the emergency department of Saudi Arabian hospitals a CDSS to deal with Acute Coronary Syndrome (ACS) is under development [14].

This system depends on evidence and involves the updates on the guidelines and prioritizes the decision according to the medical resources. The computerized CPG are evaluated by comparing the recommendations provided by the system with physicians' decision [14]. This system gives emergency physicians the ability to improve their practices and assists them to deal with life threatening diseases and provides accurate decision in real time to save patients' life.

Another example of CDSS implementation in KSA is Computerized Physician Order Entry (CPOE) system at King Saud Medical Complex Hospital (KSMCH) [11]. CPOE is a computer-based system that has replaced hand written notes. An authorized personnel enters the clinical orders, prescriptions, and diagnostic tests in the system. The system also sends alerts to physicians about possible drug allergies and drug-drug interactions. At KSMCH, this system was evaluated for its effects on the performance of clinical practices [11]. CPOE in KSMC gained $80 \%$ to $90 \%$ satisfaction of physicians. The system also saved medication wastage up to $45 \%$, reduced medication transcription error to $90 \%$, provided quick alerts on potential drugs interaction and speeded up treatment to the patient [11].

\section{B. Ministry of Health's role to improve the clinical practice}

The MoH in Saudi Arabia has launched comprehensive access to UpToDate ${ }^{\circledR}$ to support evidence based clinical decisions. This contribution of $\mathrm{MoH}$ can help Saudi Arabian clinicians to get answers of clinical problems anywhere, anytime [4]. UpToDate ${ }^{\circledR}$ is an evidence-based clinical decision system that have the most recent and reliable medical information and recommendations that will improve the care of patient and quality of hospital performance. It indeed saves the clinicians' time by producing accurate and quick clinical answers at point of care. It has more than 10,000 topics about clinical problems [5]. This service, available for free to all clinicians throughout the kingdom, will improve the clinical practices in Saudi Arabian hospitals.

\section{CONCLUSION}

CDSS aids physicians in making a decision and has a significant role in patient safety. It also increases the quality of care in health sector. In our research, we display the great impact of CDSS in healthcare and the most important challenges related to CDSS implementation including financial, ethical, technical, and social issues. We have also discussed the requirements for successful CDSS implementation. For this purpose, we have divided it into three categories, 1) CDSS content and knowledge, 2) incorporating CDSS with HIS, 3) the quality of advice provided by CDSS. Then we discussed the implementation of CDSS in Saudi Arabia as a case study by reviewing some examples and explaining the role of health min istry to support CDSS in health organizations. Despite of some weaknesses CDSS has evolved as a powerful tool to increase the quality of care in health sector. We hope that new generations of CDSS will address the challenges discussed here and in previous researches and will have greater capability and usability.

\section{REFERENCES}

[1] H. Shin and M. Markey, 'A machine learning perspective on the development of clinical decision support systems utilizing mass spectra of blood samples', Journal of Biomedical Informatics, 39(2):227-248, 2006.

[2] R. Thomson, 'Clinical Decision Support Systems', Openclinical.org, 2015.2 Available: http://www.openclinical.org/dss.html. [Accessed: 07Dec- 2015].

[3] K. Wagholikar, V. Sundararajan and A. Deshpande, 'Modeling Paradigms for Medical Diagnostic Decision Support: A Survey and Future Directions', J Med Sys, 36(5):3029-3049, 2011.

[4] Health awareness. Available: http://www.moh.gov.sa/en /HealthAwareness/Pages/UpToDate.aspx. [Accessed: 09Dec- 2015].

[5] Uptodate.

Available: http://www.uptodate.com/contents/search. [Accessed: 09Dec- 2015].

[6] D. Sittig, A. Wright, J. Osheroff, B. Middleton, J. Teich, J. Ash, E. Campbell and D. Bates, 'Grand challenges in clinical decision support', Journal of Biomedical Informatics, 41(2):387-92, 2008.

[7] M. Jaspers, M. Smeulers, H. Vermeulen and L. Peute, 'Effects of clinical decision-support systems on practitioner performance and patient outcomes: a synthesis of high-quality systematic review findings', Journal of the American Medical Informatics Association, 18(3):327-334, 2011.

[8] J. F. Sucher, F. A. Moore, S. R. Todd, R. M. Sailors, and B. A. McKinley, 'Computerized clinical decision support: a technology to implement and validate evidence based guidelines', The Journal of Trauma, 64(2), 520-537, 2008.

[9] B. Mollon, J. J. Chong, A. M. Holbrook, M. Sung, L. Thabane, and G. Foster, 'Features predicting the success of computerized decision support for prescribing: a 
systematic review of randomized controlled trials', BMC Medical Informatics and Decision Making, 9(11), 2009.

[10] J. A. Lyman, W. F. Cohn, M. Bloomrosen, and D. E. Detmer, 'Clinical decision support: progress and opportunities', Journal of the American Medical Informatics Association, 17(5), 487-492, 2010.

[11] S. Alwakeel and D. Barakah, 'Impact of CPOE on Physicians and Dentists' Work Performance at King Saud Medical Complex Hospital: A Case Study', http://hdl.handle.net/123456789/15106, 2009.

[12] M. Khalifa, 'Clinical Decision Support: Strategies for Success', Procedia Computer Science, 37, 422-427, 2014.

[13] M. Khalifa and O. Alswailem, 'Clinical Decision Support Knowledge Management: Strategies for Success', Studies in Health Technology and Informatics, 213:67-70, 2015.

[14] M. Omaish, S. Abidi and S. Abidi, 'Ontology-based computerization of Acute Coronary Syndrome clinical guideline for decision support in the emergency department', Studies in Health Technology and Informatics, 180:437-41, 2015.

[15] K. Kawamoto, 'Improving clinical practice using clinical decision support systems: a systematic review of trials to identify features critical to success', BMJ, 330(7494):765$770,2005$.

[16] M. Jaspers, M. Smeulers, H. Vermeulen and L. Peute, 'Effects of clinical decision-support systems on practitioner performance and patient outcomes: a synthesis of high-quality systematic review findings', Journal of the American Medical Informatics Association, 18(3):327-334, 2011.

[17] P. Casimir, 'Role of Clinical Decision Support Systems in Improving Clinical Practice', MOJ Clinical \& Medical Case Reports, 2(6), 2015.

[18] D. F. Doolan, D. W. Bates, and B. C. James, 'The use of computers for clinical care: a case series of advanced U.S. sites', J Am Med Inform Assoc. 10:94-107, 2003.

[19] D. F. Doolan, and D. W. Bates, 'Computerized physician order entry sy stems in hospitals: mandates and incentives', Health Aff. 21:180-188, 2002.

[20] N. Kucher, S. Koo, R. Quiroz, et al., 'Electronic alerts to prevent venous thromboembolism among hospitalized patients', N Engl J Med, 352:969-977, 2005.

[21] E. Berner and T. La Lande, 'Overview of Clinical Decision Support Sy stems, in Eta S. Berner EdD, FACMI, FHIMSS (eds) 'Clinical Decision Support Systems' Springer New York, 3-22, 2007.

[22] E. S. Berner, R. S. Maisiak, S. T. Phelan, J. I. Kennedy, G R. Heudebert and K. R. Young, 'Use of a diagnostic decision support system by internal medicine residents', Unpublished study, 2002.

[23] K. Kawamoto, C. A. Houlihan, E. A. Bala and D. F. Lobach, 'Improving clinical practice using clinical decision support systems: a systematic review of trials to identify features critical to success', BMJ 330:765, 2005.

[24] L. Jiang, B. Xu, C. Xie and H. Cai, 'A framework of emergency clinical decision support system based on MDA and resource model', International Conference on Computer Supported Cooperative Work in Design, 2014.

[25] P. Esmaeilzadeh, M. Sambasivan, N. Kumar and H. Nezakati, 'Adoption of clinical decision support systems in a developing country: Antecedents and outcomes of physician's threat to perceived professional autonomy', International Journal of Medical Informatics, 84(8):548560, 2015.

[26] E. Sanchez, C. Toro, A. Artetxe, M. Graña, C. Sanin, E. Szczerbicki, E. Carrasco and F. Guijarro, 'Bridging challenges of clinical decision support systems with a semantic approach. A case study on breast cancer', Pattern Recognition Letters, 34(14):1758-1768, 2013.

[27] E. Berner, 'Ethical and legal issues in the use of health information technology to improve patient safety', HEC Forum, 20(3):243-258, 2008.

[28] K. W. Goodman, 'Ethical and legal issues in decision support', in Eta S. Berner EdD, FACMI, FHIMSS (eds) 'Clinical Decision Support Systems' Springer New York, 126-139, 2007.

[29] Electronichealthreporter.com, '7 Things to consider when choosing clinical decision support software - Electronic Health Reporter', Available: http://electronichealthreporter.com/7-choosing-clinicaldecision-software/ [Accessed: 06- Dec- 2015].

[30] C. Klein, 'Changing before our eyes: an evolution of clinical decision support in healthcare', Ntst.com, Available: http://www.ntst.com/wp/Netsmart Whitepaper Content/13 [Accessed: 08- Dec- 2015].

[31] E. Berner, 'Informatics challenges for the impending patient information explosion', Journal of the American Medical Informatics Association, 12(6):614-617, 2005.

[32] A. Nasser, H.Zaied, M. Elmogy and S. Abd Elkader, 'Electronic health records: applications, techniques and challenges', International Journal of Computer Applications, 119(14):38-49, 2015.

[33] N. Menachemi and Collum, 'Benefits and drawbacks of electronic health record systems', RMHP, 47, 2011.

[34] Wikipedia, 'Clinical decision support system', Available: https://en.wikipedia.org/wiki/Clinical_decision_support_s ystem [Accessed: 08- Dec- 2015].

[35] K. T. Win, W. Susilo, and Y. Mu, 'Personal health record systems and their security protection', Journal of medical sy stems, 30(4):309-315, 2006.

[36] E. S. Berner, R. S. Maisiak, G. R. Heudebert, K. R. Young, 'Clinician performance and prominence of diagnoses displayed by a clinical diagnostic decision support system', Proc AMIA Fall Symp., 76-80, 2003.

[37] R. N. Schiffman, C. Brandt, Y. Liaw, et al. 'A design model for computer-based guideline implementation based on information management services', J Am Med Inform Assoc. 6:99-103, 1999.

[38] J. M. Teich, J. A. Osheroff, E. A. Pifer, D. F. Sittig, R. A. Jenders, 'The CDS Expert Review Panel. Clinical decision support in electronic prescribing: recommendations and an action plan', J Am Med Inform Assoc. 12:365-76, 2005.

[39] R. N. Charette, 'EHRs: electronic health records or exceptional hidden risks?', Commun. ACM, 49(6):120, 2006.

[40] E. S. Berner, 'Ethical and legal issues in the use of health information technology to improve patient safety'. HEC Forum 20.3: 243-258, 2008.

[41] K. W. Goodman, 'Ethical and legal issues in decision support', in Eta S. Berner EdD, FACMI, FHIMSS (eds) 'Clinical Decision Support Systems' Springer New York, 126-139, 2007.

[42] L. Forrow, S. A. Wartman and D. W. Brock, 'Science, ethics, and the making of clinical decisions', JAMA 259:3161-3167, 1988.

[43] K. W. Goodman, 'Outcomes, futility, and health policy research, In: Goodman KW, ed. Ethics, computing and medicine: informatics and the transformation of healthcare. Cambridge and New York: Cambridge University Press, 116-138, 1997.

[44] R. A. Miller and K. W. Goodman, 'Ethical challenges in the use of decision-support software in clinical practice. In: Goodman KW, ed. Ethics, computing and medicine: 
informatics and the transformation of healthcare. Cambridge and New York: Cambridge University Press; 1997:102-115.

[45] N. P. Terry, 'When the machine that goes 'ping' causes harm: default torts rules and technologically-mediated healthcare injuries. St. LouisUniversity Law Journal, 46:37, 2002.

[46] J. M. Sorace, J. J. Berman, G. E. Carnahan and G. W. Moore, 'PRELOG: precedence logic inference software for blood donor deferral', Proc Annu Symp Comput Appl Med Care, 976-977, 1991.

[47] S. Subramanian, 'Creating Knowledge-Based Clinical Decision Support System Using Cloud Computing Architecture', ProQuest, 2013.

[48] M. Abumelha, A. Hashbal, F. Nadeem, N. Aljohani, 'Development of Infection Control Surveillance System for Intensive Care Unit: Data Requirements and Guidelines', International Journal of Intelligent Systems and Applications, 8(6): 19-26, 2016.

\section{Authors' Profiles}

Sahar S. Alqahtani: Post-graduated student for master degree in information systems at King Abdulaziz University. She is teacher assistant in King Khalid University. She has published 2 papers in international journals.

Sabah Alshahri: Post-graduated student for master degree in information systems at King Abdulaziz University. She is teacher assistant in King Khalid University. She has published 2 papers in international journals.

Ahood I. Almaleh: Post-graduate student for master degree for information systems at King Abdulaziz University. She is teacher assistant in Najran University. She has published 2 papers in international journals.

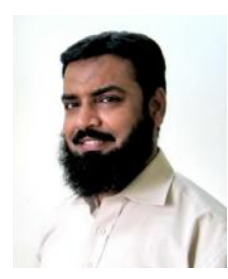

Farrukh Nadeem received his Ph.D. in 2009 from the University of Innsbruck, Austria. Since July 2009, he has been working as an Assistant Professor. His main research interests include performance modeling and prediction, and scheduling scientific workflows in distributed systems, particularly the Grid and the Cloud. He has been involved in a several Austrian and Saudi research and development projects. Farrukh is author of more than 24 papers, including four book chapters. He holds several distinctions and awards during his educational career.

How to cite this paper: Sahar S. Alqahtani, Sabah Alshahri, Ahood I. Almaleh, Farrukh Nadeem, "The Implementation of Clinical Decision Support System: A Case Study in Saudi Arabia", International Journal of Information Technology and Computer Science (IJITCS), Vol.8, No.8, pp.23-30, 2016. DOI: 10.5815/ijitcs.2016.08.03 